\title{
A meta-analysis and systematic review of the therapeutic effects of arthroscopy combined with intra-articular injection of sodium hyaluronate in the treatment of knee osteoarthritis
}

\author{
Xiao Yang ${ }^{1,2 \#}$, Weimin Liang ${ }^{1,2 \#}$, Jiabing $\mathrm{Li}^{1,2}$, Pan Liu ${ }^{1,2}$ \\ ${ }^{1}$ Department of Orthopedics, Sichuan Provincial People's Hospital, University of Electronic Science and Technology of China, Chengdu, China; \\ ${ }^{2}$ Chinese Academy of Sciences Sichuan Translational Medicine Research Hospital, Chengdu, China \\ Contributions: (I) Conception and design: X Yang, W Liang; (II) Administrative support: J Li; (III) Provision of study materials or patients: X Yang, \\ W Liang, P Liu; (IV) Collection and assembly of data: All authors; (V) Data analysis and interpretation: X Yang, J Li, P Liu; (VI) Manuscript writing: \\ All authors; (VII) Final approval of manuscript: All authors. \\ \#These authors contributed equally to this work. \\ Correspondence to: Jiabing Li; Pan Liu. No. 32, West Section 2, First Ring Road, Chengdu, Sichuan Provincial People's Hospital, University of \\ Electronic Science and Technology of China, Chengdu, China. Email: sports_medicine@126.com; Lliupan126L@126.com.
}

\begin{abstract}
Background Knee osteoarthritis (KOA) causes joint pain and dysfunction that severely affects the patient's ability to walk. Arthroscopy combined with intraarticular injection of sodium hyaluronate can slow the development of knee osteoarthritis, but there is a lack of rigorous evidence-based medicine evaluation, and it has not been recognized by some scholars at home and abroad. This paper analyzed the clinical efficacy of arthroscopy combined with intraarticular injection of sodium hyaluronate in slowing knee osteoarthritis based on Lysholm score.
\end{abstract}

Methods: Randomized control trials of arthroscopy combined with intra-articular injection of sodium hyaluronate in the treatment of KOA on Chinese and English databases were retrieved using the following search terms: "knee osteoarthritis", "arthroscopic debridement of the joint", "articular cavity", and "sodium hyaluronate". The quality of the literature was evaluated using the Rev Man 5.3 software.

Results: Twelve literatures were included in the meta-analysis. Regarding Newcastle-Ottawa Scale (NOS) score, of the 12 literatures, 7 literatures (58.33\%) scored 6-9 points, 3 literatures (25\%) scored 3-5 points, and 2 literatures (16.67\%) scored 0-2 points. With a random effects model used for analysis, the Lysholm score after 1 month of combined treatment was significantly higher than that before treatment [mean difference $(\mathrm{MD})=30.65$, 95\% confidence interval $(\mathrm{CI}): 19.44,41.86, \mathrm{P}<0.01]$. Further, after 3 months of combination treatment, the Lysholm score was significantly higher than that before treatment $(\mathrm{MD}=24.04$, 95\% CI: $13.75,34.32, \mathrm{P}<0.01)$, and the same trend was also observed after 6 months $(\mathrm{MD}=20.41,95 \%$ CI: $14.43,26.40, \mathrm{P}<0.01$ ) and after 12 months ( $\mathrm{MD}=20.86,95 \% \mathrm{CI}: 8.99,32.72, \mathrm{P}<0.01$ ) of combination treatment. Overall, the combined treatment achieved significantly better clinical therapeutic effects than the single treatments in the control group [odds ratio $(\mathrm{OR})=7.51,95 \% \mathrm{CI}: 4.84,11.65, \mathrm{P}<0.01$ ].

Discussion: This meta-analysis has confirmed that arthroscopy combined with intra-articular injection of sodium hyaluronate demonstrates significant clinical therapeutic effects in the treatment of KOA. This combination treatment can significantly improve knee joint function, relieve clinical symptoms, and improve patients' quality of life.

Keywords: Arthroscopy; intra-articular injection of sodium hyaluronate; knee osteoarthritis (KOA); therapeutic effects

Submitted Jul 21, 2021. Accepted for publication Sep 02, 2021.

doi: 10.21037/apm-21-2145

View this article at: https://dx.doi.org/10.21037/apm-21-2145 


\section{Introduction}

Knee osteoarthritis (KOA) is a common chronic orthopedic joint disease which entails non-inflammatory degeneration of the articular cartilage of the knee. It arises from the wear and disappearance of articular cartilage over time, commonly known as bone spurs and bone hyperplasia (1). Causes of KOA include chronic strain, obesity, osteoporosis, trauma, and genetic factors. KOA mainly manifests as joint pain, swelling, and restricted movement, and in severe cases, joint deformities may develop, affecting the individual's quality of life (2).

Generally, surgery is required when conservative treatment proves ineffective. Surgical plans vary depending on the patient's condition and can include arthroscopic joint debridement surgery, osteotomy, unicondylar knee arthroplasty, and total artificial knee replacement surgery (3).

Arthroscopic joint debridement includes synovectomy, chondroplasty, the removal of free objects, meniscus resection, and loosening of the ligaments (4). It can not only aid in reducing the release of inflammatory factors in the joint cavity, but can also relieve various clinical symptoms such as pain and edema in the joint. Although arthroscopic joint debridement cannot completely restore the anatomical structure of the joint, it can repair diseased tissue and remove inflammatory mediators. As a minimally invasive surgery, arthroscopic joint debridement reduces the patient's time in the operating room, and minimizes blood loss and tissue damage. It also helps to minimize pain during healing, speeds up recovery, and reduces the chance of complications (5).

The knee joint cavity is formed by approximately $3-$ $5 \mathrm{~mL}$ synovial fluid, together with articular cartilage (6). Hyaluronic acid is an important component of normal joint synovial fluid that helps to prevent wear and tear of cartilage. It is a polymer comprising molecular units which, at most, consists of 25,000 units. It is mainly synthesized by synovial cells and mononuclear phagocytes. In patients with $\mathrm{KOA}$, the production and quality of hyaluronic acid cannot be guaranteed, but supplementation of hyaluronic acid in vitro makes it possible to relieve friction and pain $(7,8)$. In general, two weeks after arthroscopy, the exudation caused by surgical trauma will stop. At this time, the poor effusion can be extracted by puncture, and the supplementation of exogenous sodium hyaluronate can improve the content of sodium hyaluronate in the synovial membrane, and re-form a natural barrier to prevent further destruction and disappearance of cartilage matrix. Therefore, the biological function of synovial membrane is improved under pathological condition, joint friction and pain are relieved or eliminated. By inhibiting leukocyte movement and chemotaxis, it can reduce synovial permeability, increase the production of high molecular sodium hyaluronate, and reduce intraarticular exudation. Covering and protecting nociceptors, pain mediators are combined to relieve pain. It binds to glycoproteins and prevents them from participating in the inflammatory process. Meanwhile, sodium hyaluronate enters the cartilage matrix and forms polymers with glycoproteins to repair damaged cartilage. Arthroscopic surgery has the characteristics of precision, short recovery time, and few complications, and combined with intraarticular injection of sodium hyaluronate can significantly enhance the clinical efficacy. Cho et al. [2011] (9) has shown that cartilage degeneration is the main pathological feature of KOA, and that intraarticular injection of exogenous sodium hyaluronate forms a protective barrier to lubricate the joint cavity, which is conducive to the repair of the articular cartilage (9).

The majority of literatures to date have been singlecenter randomized controlled trials (RCTs) with small sample sizes. In the present study, a meta-analysis was conducted to systematically analyze the effectiveness of arthroscopy combined with intra-articular injection of sodium hyaluronate in the treatment of KOA, with the aim of providing an evidence-based foundation for the clinical treatment of KOA.

Most of the existing studies are small sample size, singlecenter randomized controlled trials, and simple arthroscopy or simple intraarticular injection of sodium hyaluronate for the treatment of KOA. The innovation of this study is to compare the effectiveness of arthroscopy combined with intraarticular injection of sodium hyaluronate and arthroscopic debridement alone in the treatment of KOA by using systematic evaluation and meta-analysis, so as to provide scientific and evidence-based evidence for the clinical treatment of KOA.

We present the following article in accordance with the PRISMA reporting checklist (available at https://dx.doi. org/10.21037/apm-21-2145).

\section{Methods}

\section{Literature retrieval}

With "knee osteoarthritis" "arthroscopic debridement of the joint" "articular cavity," and "sodium hyaluronate" as 
the search terms, Chinese- and English-language databases were searched from database establishment to April 10, 2021 to identify RCTs of arthroscopy combined with intraarticular injection of sodium hyaluronate in the treatment of KOA. The databases included PubMed, Medline, Embase, Web of Sciences, Chinese Biomedical Literature Database, Wanfang Chinese Biomedical Association Digital Journals, Wanfang Digital Journals Full-text Database, and Weipu Chinese Sci-tech Journals Full-text Database.

Relevant literatures were retrieved using the Boolean logic retrieval method. The literature quality was evaluated using the Cochrane Collaboration's RevMan 5.3 software.

Some literatures were eliminated after reading of their titles and abstracts. A second screening was then performed according to the inclusion and exclusion criteria, and the literatures were traced using a search engine. A third and final screening was conducted by reading the full texts of the included literature.

\section{Inclusion and exclusion criteria}

The literatures to be meta-analyzed were selected in line with the following inclusion criteria: (I) RCTs and retrospective case-control literatures; (II) participants with KOA diagnosed according to the American Rheumatology Society (ARC) standards; (III) literatures using the Lysholm score as the research index, reported with the $95 \%$ confidence interval (CI); (IV) complete basic clinical data and observation indicators.

Literatures meeting the following criteria were excluded: (I) research on post-traumatic arthritis, post-suppurative arthritis, and other systemic diseases; (II) the participants had other types of surgery at the surgical site; (III) repeatedly published research; (IV) conference speeches, literature reviews, case study reports, lectures, and review literature.

Two senior experts performed the literature screening. Any inconsistencies were resolved by discussion or through arbitration by another expert.

\section{Data extraction}

The two experts used a unified Microsoft Excel spreadsheet to independently collate data. Any inconsistencies were resolved by discussion or through arbitration by another expert. The following data were collated: (I) the research title, research time, and type; (II) the follow-up time and the number of cases lost to follow-up; (III) the first author's name, publication year, and journal name; (IV) general information of the research participants including the Geographical region, sample size, sex ratio, and age distribution; (V) observation indicators including the Lysholm score before surgery and at 1, 3, and 6 months after surgery.

\section{Risk of bias and quality assessment}

Two researchers conducted a bias risk assessment simultaneously. Any inconsistencies were resolved by discussion or through arbitration by a third expert. In this study, the Cochrane Collaboration's bias risk assessment tool was used. The conclusion of low, unclear, or high risk of bias was reached according to the following four aspects: randomization method, blinding method, allocation concealment, and the number of cases lost to follow up.

The Newcastle-Ottawa Scale (NOS) was used to evaluate the quality of the literature on the basis of patient selection, study comparability, and outcome. Each item has a score of 1-2 points. There are 8 items in total. and the total score ranged from 0-9 points. Literatures with a score of 6-9 points were considered high-level research (low risk of bias), those with 3-5 points were considered middle-level research (unclear risk of bias), and those with $0-2$ points were considered low-level research (high risk of bias).

\section{Statistical analysis}

Stata SE12.0 (College Station, USA) was used to perform statistical analyses. RevMan 5.3 was used to assess the risk bias of the included literatures. Continuous variables were expressed as the mean difference (MD), and discontinuous variables were expressed as the odds ratio $(\mathrm{OR})$ and relative risk (RR). Each effect was expressed using the $95 \%$ CI. When $\mathrm{P}>0.01$ and $\mathrm{I}^{2}<50 \%$, the fixed effects model (FEM) was used for meta-analysis; when $\mathrm{P}<0.01, \mathrm{I}^{2}>50 \%$, the random effects model (REM) was used for meta-analysis.

\section{Results}

\section{Basic information of the included literature}

A total of 265 literatures were obtained through the database and the register. By reading the abstract and title of the articles, 63 articles were repeatedly published, 29 articles were unqualified, and 131 articles were left from the 42 articles for other reasons. By reading the 
Identification of studies via databases and registers

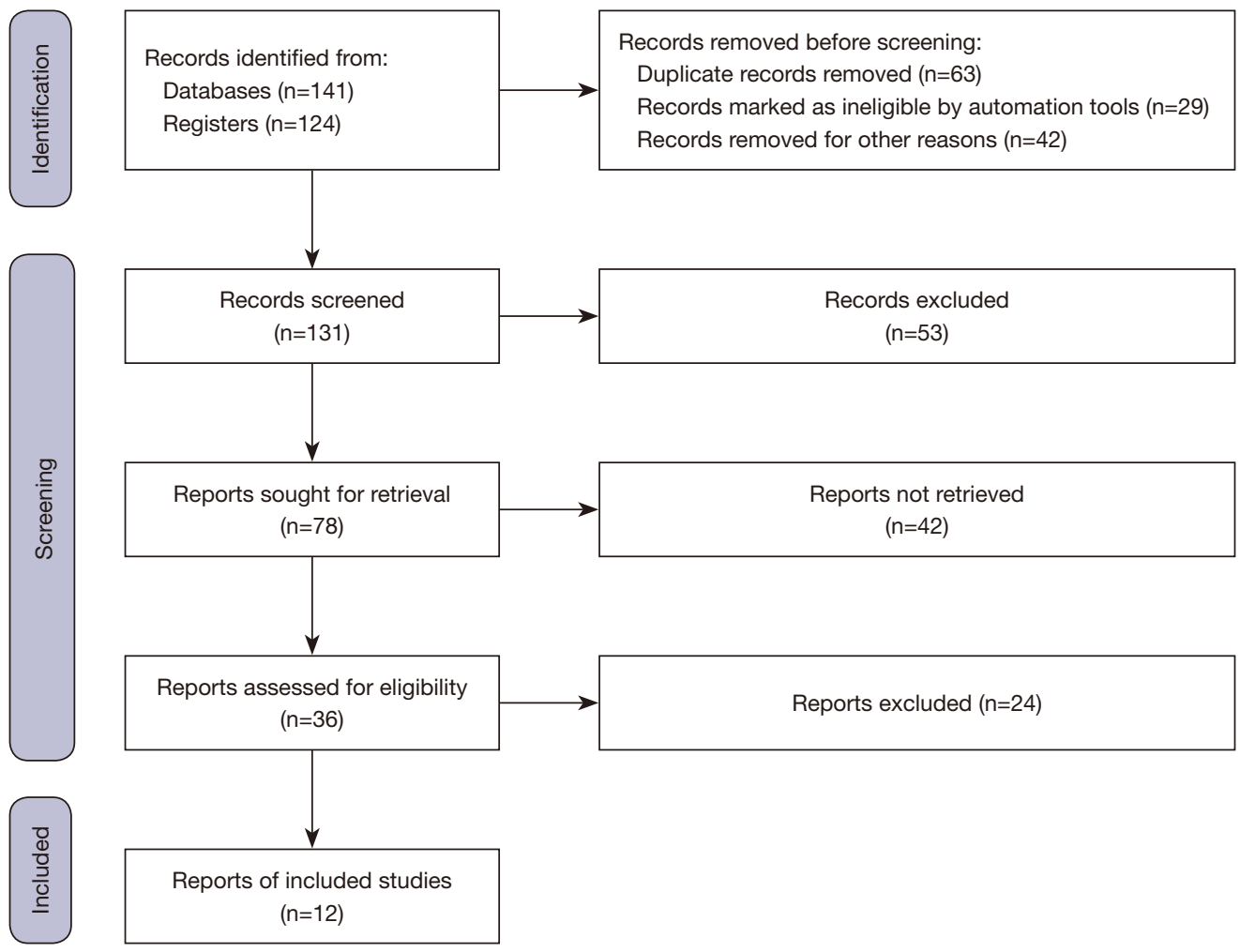

Figure 1 Flowchart of the literature selection process.

full text, 53 articles with repeated research subjects were eliminated, leaving 78 articles; 42 literatures that were not randomized controlled trials, such as review reports, were excluded, leaving 36 literatures; 24 articles that could not be extracted were excluded, and 12 articles were included in the study (Figure 1). Of the remaining 36 literatures, 24 were eliminated due to having incomplete data. Finally, 12 eligible literatures were included in the study. According to the quality evaluation results of the Newcastle-Ottawa Scale, there were 7 literatures $(58.33 \%)$ with $6-9$ points, 3 literatures (25\%) with 3-5 points, and 2 literatures (16.67\%) with $0-2$ points.

The 12 included literatures involved 751 participants. All the literatures were small-sample literatures, with their sample sizes ranging from 35 to 95 cases. All of the research participants were over 20 years of age. All 12 literatures detailed the sample size, sex ratio, age, treatment method, follow-up time, and outcome indicators. Table 1 shows the basic characteristics of the included literature.

\section{Risk of bias evaluation}

Figures 2 and 3 show the results of bias risk assessment performed using the RevMan 5.3 software. Among the 12 RCTs included, 3 (25\%) RCTs (10-12) described the correct random allocation method, 2 (16.67\%) RCTs $(13,14)$ adopted allocation concealment, and 1 RCT (8.33\%) (15) used the blind method.

\section{Lysholm scores before surgery and at 1 month after surgery}

The forest plot in Figure 4 shows the Lysholm scores before surgery and at 1 month after surgery. Of the 12 literatures, 7 detailed the MD and standard deviation (SD) of the Lysholm scores before and at 1 month after surgery, and the heterogeneity results showed $\mathrm{I}^{2}=99 \%>50 \% \mathrm{P}<0.00001$, which indicated obvious heterogeneity. Consequently, an REM was used for analysis, and the Lysholm score after 1 month of combined treatment was found to be significantly higher than that before treatment $(\mathrm{MD}=30.65,95 \% \mathrm{CI}$ : $19.44,41.86, \mathrm{P}<0.00001)$. 


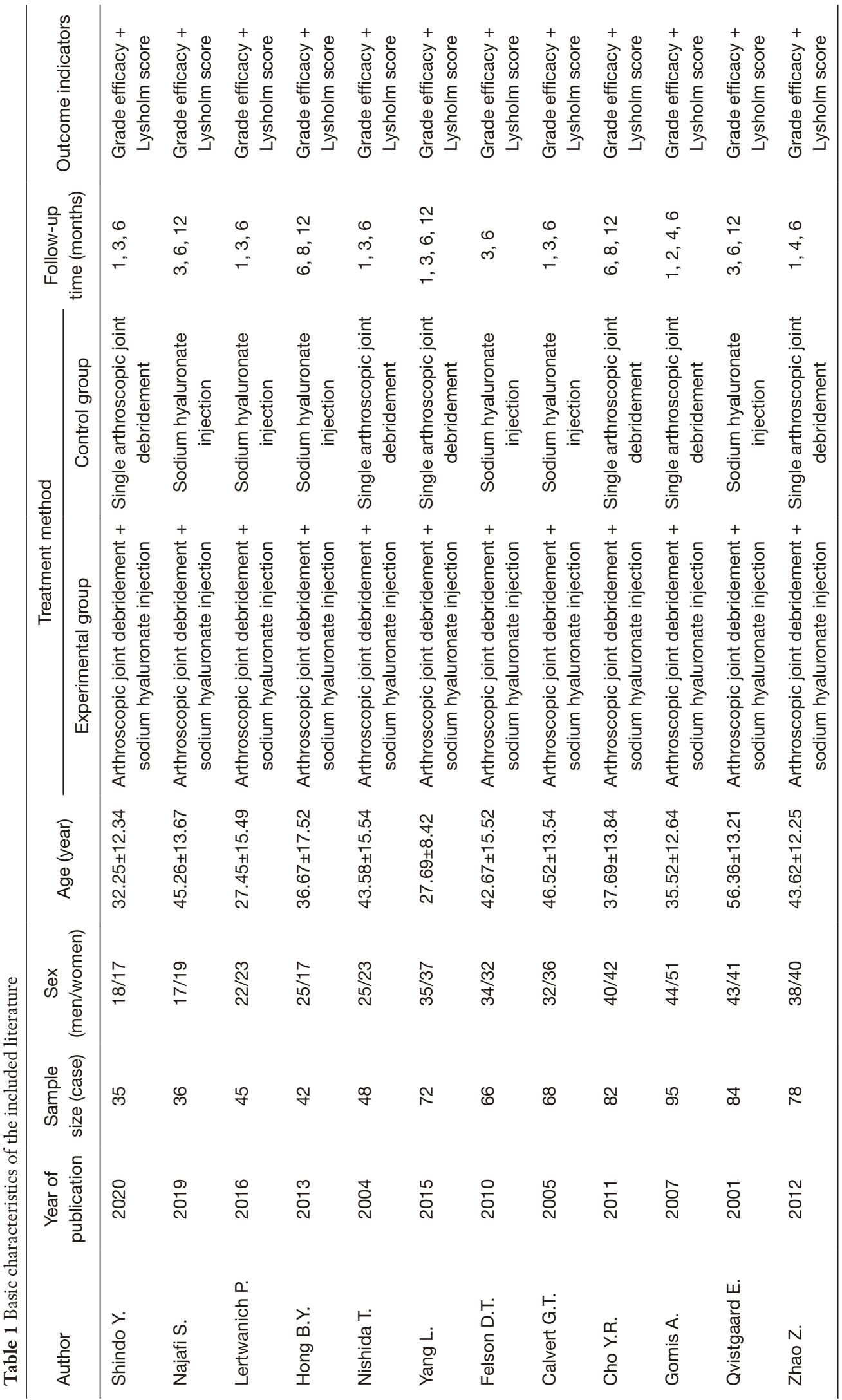




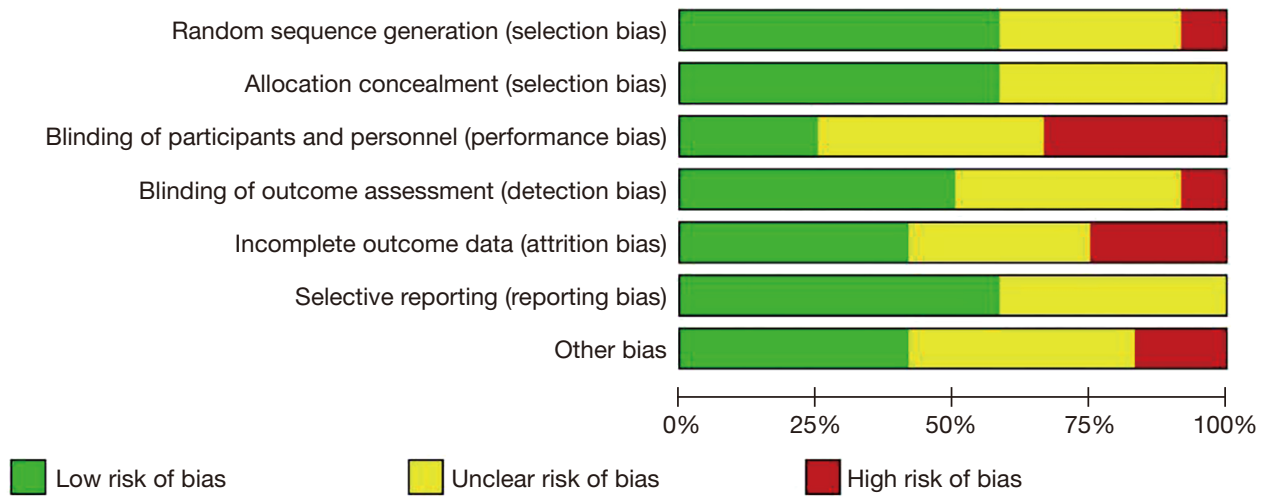

Figure 2 Risk of bias assessment of the included literature.

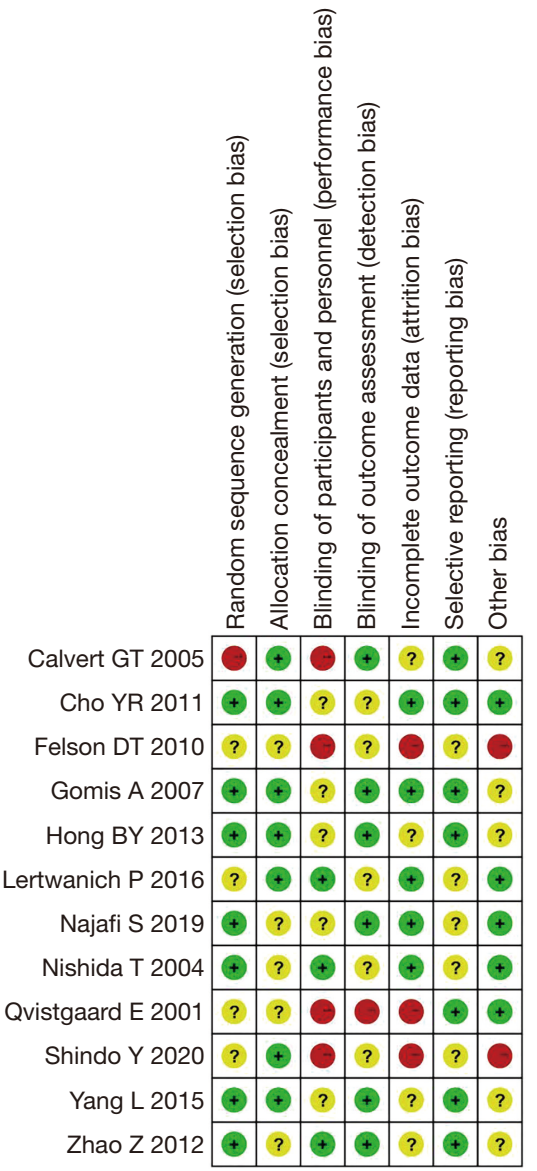

Figure 3 Individual risk of bias results for the 12 included literatures. "+" means low-risk bias; “-" means high-risk bias; "?" means unclear risk.

\section{Lysholm scores before surgery and at 3 months after surgery}

The forest plot in Figure 5 shows the Lysholm scores before surgery and at 3 months after surgery. Of the 12 literatures, 8 detailed the MD and SD of the Lysholm scores before surgery and at 3 months after surgery. The heterogeneity results showed $\mathrm{I}^{2}=99 \%>50 \%, \mathrm{P}<0.01$, which indicated obvious heterogeneity. Consequently, an REM was used for analysis, and the Lysholm score after 3 months of combined treatment was found to be significantly higher than that before treatment $(\mathrm{MD}=24.04,95 \% \mathrm{CI}: 13.75,34.32, \mathrm{P}<0.00001)$.

\section{Lysholm scores before surgery and at 6 months after surgery}

The forest plot in Figure 6 shows the Lysholm scores before surgery and at 6 months after surgery. All of the 12 literatures detailed the MD and SD of the Lysholm scores before surgery and at 6 months after surgery. The heterogeneity results showed $\mathrm{I}^{2}=98 \%>50 \%, \mathrm{P}<0.00001$, which indicated obvious heterogeneity. Consequently, an REM was used for analysis, and the Lysholm score after 6 months of combined treatment was found to be significantly higher than that before treatment $(\mathrm{MD}=20.41,95 \% \mathrm{CI}: 14.43,26.40, \mathrm{P}<0.00001)$.

\section{Lysholm scores before surgery and at 12 months after surgery}

The forest plot in Figure 7 shows the Lysholm scores 


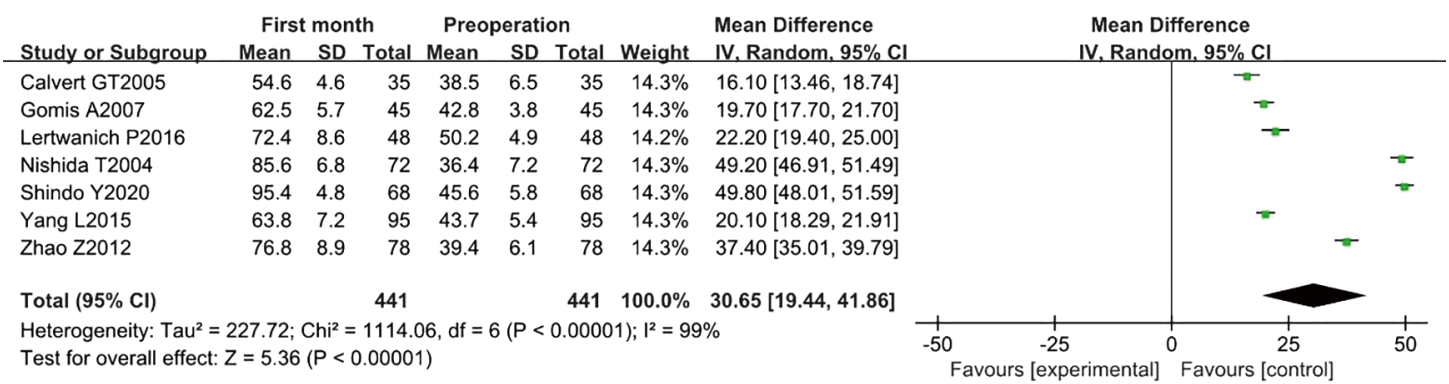

Figure 4 Forest plot showing the Lysholm scores before surgery and at 1 month after surgery.

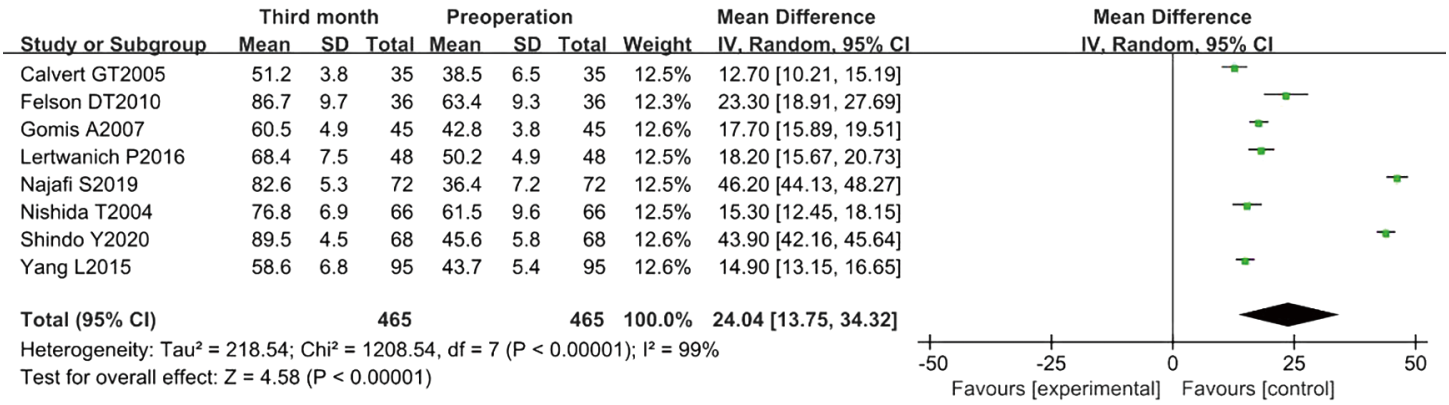

Figure 5 Forest plot showing the Lysholm scores before surgery and at 3 months after surgery.

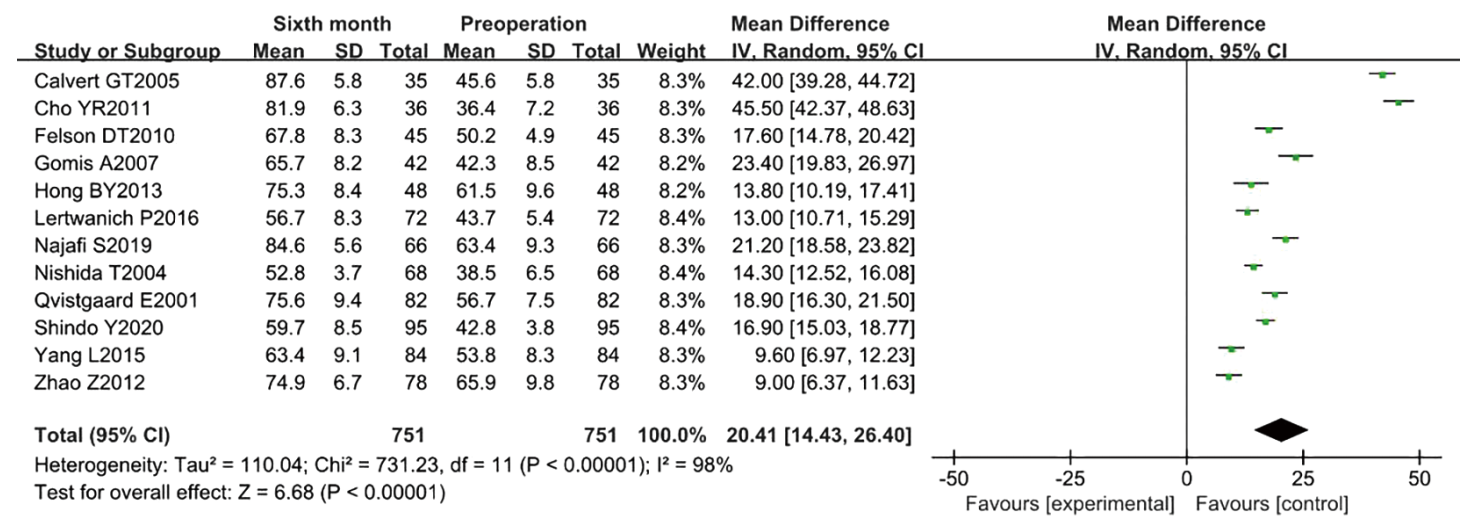

Figure 6 Forest plot showing the Lysholm scores before surgery and at 6 months after surgery.

before surgery and at 12 months after surgery. Of the 12 literatures, 5 detailed the MD and SD of the Lysholm scores before surgery and at 12 months after surgery. The heterogeneity results showed $\mathrm{I}^{2}=99 \%>50 \%, \mathrm{P}<0.00001$, which indicated obvious heterogeneity. Consequently, an REM was used for analysis. The Lysholm score after 12 months of combined treatment was found to be significantly higher than that before treatment $(M D=20.86$,
95\% CI: 8.99, 32.72, P=0.0006).

\section{Clinical efficacy in the experimental and control groups}

Figure 8 shows the clinical efficacy of the treatments in the experimental group and the control group. All 12 literatures detailed the clinical efficacy of the experimental group and control group treatments. The heterogeneity results showed 


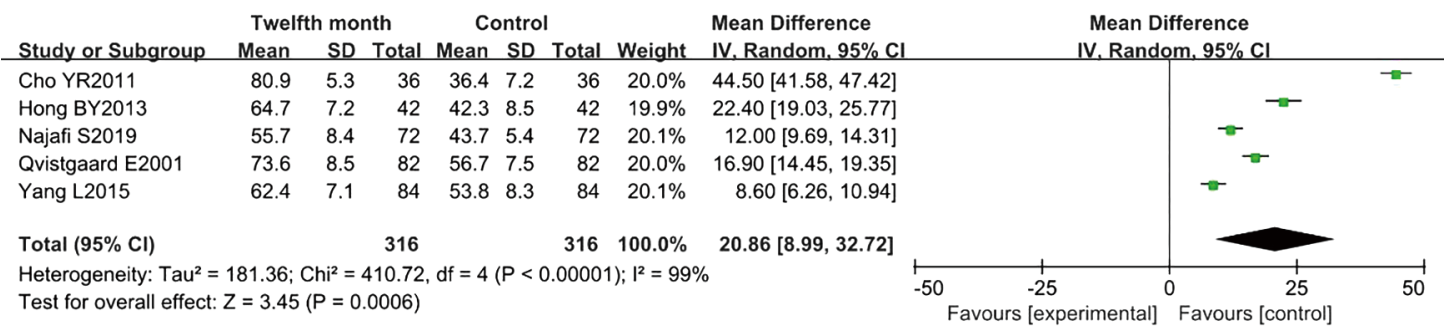

Figure 7 Forest plot showing the Lysholm scores before surgery and at 12 months after surgery.

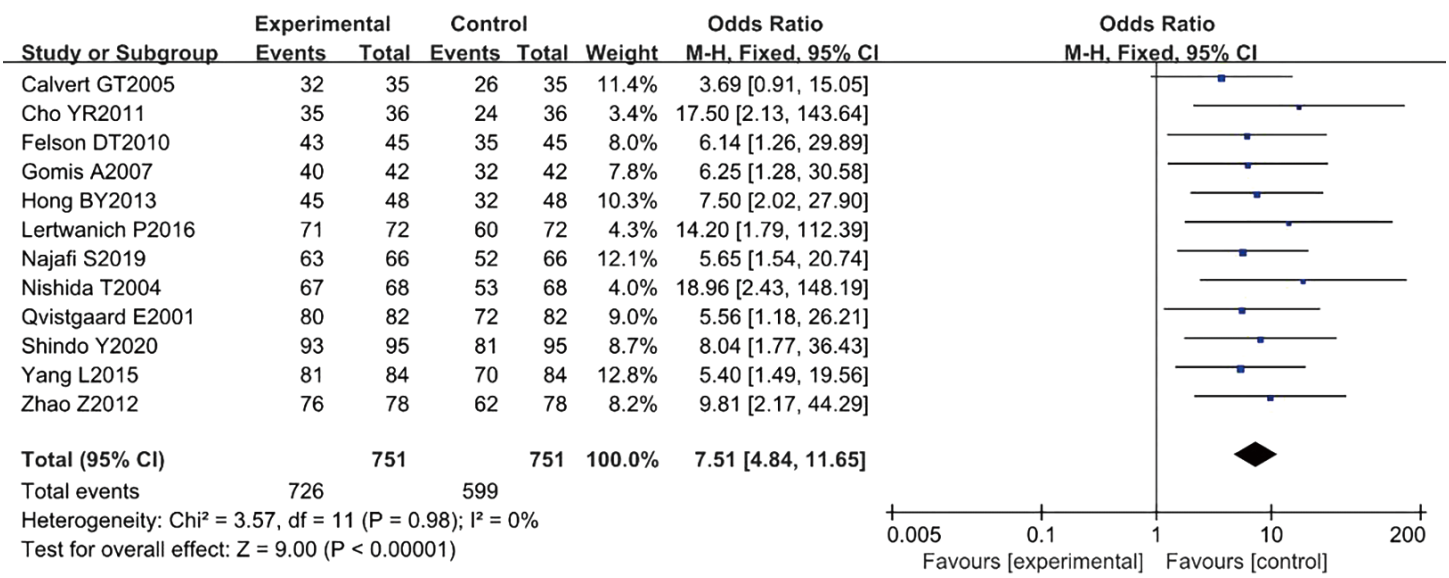

Figure 8 Forest plot showing the clinical efficacy of the treatments in the experimental group and the control group.

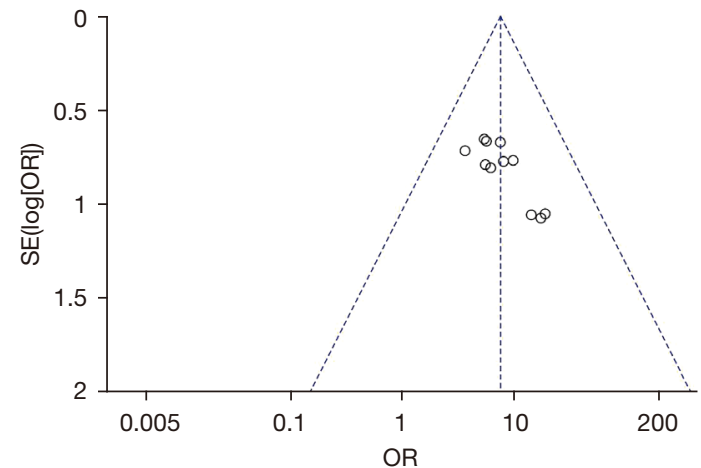

Figure 9 Funnel chart of the publication bias of the included literature. The funnel chart indicates that the included literature is reliable and that bias is not a key factor affecting the conclusion.

$\mathrm{I}^{2}=0 \%<50 \%, \mathrm{P}=0.98$, which indicated no heterogeneity. Consequently, an FEM was used for analysis, and the clinical efficacy of the combined treatment was found to be significantly better than that of the single treatments in the control group (OR =7.51, 95\% CI: 4.84, 11.65, $\mathrm{P}<0.00001)$.

\section{Publication bias results}

Figure 9 shows a funnel plot of the publication bias of the clinical efficacy data in the included literature. In the funnel chart, the circles of most literatures were basically symmetrical along the center line, which indicated there to be no publication bias and that the results obtained were credible.

\section{Discussion}

This meta-analysis of 12 literatures was conducted to evaluate the therapeutic effects of arthroscopy combined with intra-articular injection of sodium hyaluronate in the treatment of KOA. The quality of the included literature was evaluated using the NOS. Of the 12 included literatures, 7 literatures $(58.33 \%)$ scored 6-9 points, 3 literatures (25\%) scored $3-5$ points, and 2 (16.67\%) scored 0-2 points. Of the 12 RCTs, 3 (25\%) RCTs described the correct random allocation method, 2 (16.67\%) RCTs adopted allocation concealment, and 1 (8.33\%) reference 
used the blind method (16).

Arthroscopic joint debridement can form a fibrin clot to cover the cartilage surface at the same time as removing degenerative and necrotic articular cartilage. A syringe was used to inject $0.9 \%$ sodium chloride and normal saline to clean the articular cavity and completely eliminate the necrotic cartilage debris can eliminate necrotic cartilage debris and significantly improve the intra-articular environment and joint function. Additionally, arthroscopic joint debridement completely removes the inflammatory mediators that cause osteoarthritis, which is conducive to the normal secretion of joint fluid (17). However, arthroscopic joint debridement can also lead to the loss of intra-articular synovial fluid, in which the level of sodium hyaluronate decreases (18). Supplementation of exogenous sodium hyaluronate can not only protect the tissue structure in the joint cavity, but can also induce the secretion of sodium hyaluronate by synovial cells in the bone joint, which in turn binds to glycoproteins in the synovial fluid, reducing the release of inflammatory factors. Sodium hyaluronate mainly acts as a lubricating barrier and pressure buffer in the joint cavity, and can protect articular cartilage and repair damaged cartilage (19). Sodium hyaluronate can effectively prevent cell apoptosis in knee joint cavity and control fibrosis, so as to reduce pain. However, it has little effect on the knee joint which has been degraded and damaged. It is made up of the patient's own venous blood platelet rich plasma after centrifugal getting rich in a variety of growth factors, it can effectively improve cartilage matrix generation function, promote cartilage cells, inhibit osteoclast synthesis, improve the knee stability, optimize the function of the knee joint. At the same time, platelet-rich plasma comes from the patient's own plasma, which can avoid rejection and has high safety. However, the long-term efficacy of platelet rich plasma is not obvious, and the efficacy of intraarticular injection of platelet rich plasma is not better than that of arthroscopy combined with intraarticular injection of sodium hyaluronate.

The Lysholm Knee Scale comprises eight items measuring pain (30 points), instability (30 points), thigh atrophy (5 points), swelling (10 points), lameness (5 points), stair climbing (10 points), squatting posture (5 points), and use of support (5 points), with the total score ranging from 0 to 100 points. The meta-analysis showed that the Lysholm scores at 1, 3, 6, and 12 months after the combined treatment were statistically significantly better than those before treatment $(\mathrm{P}<0.05)$. This observation suggested that exercise increases the muscle strength in the affected area, and the stability of the knee joint is significantly improved after the operation, which is consistent with the results of Tammachote et al.'s study (20).

Further, the clinical efficacy of arthroscopy combined with intra-articular injection of sodium hyaluronate was significantly better than that of the single treatments in the control group $(\mathrm{P}<0.05)$, suggesting that this combination treatment can improve knee joint function and relieve patients' clinical symptoms (21). The funnel chart of publication bias showed there to be no publication bias in the included literatures; thus, the conclusions obtained were credible and bias was not a major factor affecting the results.

\section{Conclusions}

This meta-analysis has shown that arthroscopy combined with intra-articular injection of sodium hyaluronate demonstrates significant clinical effects in the treatment of KOA. It can significantly improve knee joint function, relieve clinical symptoms, and improve patients' quality of life. The main limitations of this study include the small number of included literatures and the differences in criteria for efficacy evaluation, resulting in insufficient analysis results. In future, multi-center and large-scale clinical literatures are needed for further research. In conclusion, this study provides a scientific basis for the treatment of KOA.

\section{Acknowledgments}

Funding: Sichuan Provincial Department of Science and Technology (2019YFS0318).

\section{Footnote}

Reporting Checklist: The authors have completed the PRISMA reporting checklist Available at https://dx.doi. org/10.21037/apm-21-2145

Conflicts of Interest: All authors have completed the ICMJE uniform disclosure form (available at https://dx.doi. org/10.21037/apm-21-2145). The authors have no conflicts of interest to declare.

Ethical Statement: The authors are accountable for all aspects of the work in ensuring that questions related to the accuracy or integrity of any part of the work are appropriately investigated and resolved. 
Open Access Statement: This is an Open Access article distributed in accordance with the Creative Commons Attribution-NonCommercial-NoDerivs 4.0 International License (CC BY-NC-ND 4.0), which permits the noncommercial replication and distribution of the article with the strict proviso that no changes or edits are made and the original work is properly cited (including links to both the formal publication through the relevant DOI and the license). See: https://creativecommons.org/licenses/by-nc$\mathrm{nd} / 4.0 /$.

\section{References}

1. Madara K, Aljehani M, Pozzi F, et al. The effect of extension assist orthosis with pneumatic bladders on pain and function for patients with early knee osteoarthritis. Ann Transl Med 2019;7:S247.

2. Najafi S, Sanati E, Khademi M, et al. Intra-articular botulinum toxin type A for treatment of knee osteoarthritis: Clinical trial. Toxicon 2019;165:69-77.

3. Lertwanich P, Lamsam C. Efficacy of a Single IntraArticular Injection of 2\% Sodium Hyaluronate Plus 0.5\% Mannitol in Patients with Symptomatic Osteoarthritis of the Knee: A Preliminary Report. J Med Assoc Thai 2016;99:1094-101.

4. Hong BY, Lim SH, Im SA, et al. Effects of acute joint effusion on balance in patients with knee osteoarthritis. Am J Phys Med Rehabil 2013;92:45-52.

5. Nishida T, Kubota S, Kojima S, et al. Regeneration of defects in articular cartilage in rat knee joints by CCN2 (connective tissue growth factor). J Bone Miner Res 2004;19:1308-19.

6. Yang L, Zhang J, Wang G. The effect of sodium hyaluronate treating knee osteoarthritis on synovial fluid interleukin $-1 \beta$ and clinical treatment mechanism. Pak J Pharm Sci 2015;28:407-10.

7. Felson DT. Arthroscopy as a treatment for knee osteoarthritis. Best Pract Res Clin Rheumatol 2010;24:47-50.

8. Calvert GT, Wright RW. The use of arthroscopy in the athlete with knee osteoarthritis. Clin Sports Med 2005;24:133-52.

9. Cho YR, Hong BY, Lim SH, et al. Effects of joint effusion on proprioception in patients with knee osteoarthritis: a single-blind, randomized controlled clinical trial. Osteoarthritis Cartilage 2011;19:22-8.

10. Gomis A, Miralles A, Schmidt RF, et al. Nociceptive nerve activity in an experimental model of knee joint osteoarthritis of the guinea pig: effect of intra-articular hyaluronan application. Pain 2007;130:126-36.

11. Qvistgaard E, Kristoffersen H, Terslev L, et al. Guidance by ultrasound of intra-articular injections in the knee and hip joints. Osteoarthritis Cartilage 2001;9:512-7.

12. Zhao Z, Ji H, Jing R, et al. Extracorporeal shock-wave therapy reduces progression of knee osteoarthritis in rabbits by reducing nitric oxide level and chondrocyte apoptosis. Arch Orthop Trauma Surg 2012;132:1547-53.

13. Tang Y, Pan ZY, Zou Y, et al. A comparative assessment of adipose-derived stem cells from subcutaneous and visceral fat as a potential cell source for knee osteoarthritis treatment. J Cell Mol Med 2017;21:2153-62.

14. Maheu E, Avouac B, Dreiser RL, et al. A single intraarticular injection of $2.0 \%$ non-chemically modified sodium hyaluronate vs $0.8 \%$ hylan G-F 20 in the treatment of symptomatic knee osteoarthritis: A 6-month, multicenter, randomized, controlled non-inferiority trial. PLoS One 2019;14:e0226007.

15. Cooper C, Rannou F, Richette P, et al. Use of Intraarticular Hyaluronic Acid in the Management of Knee Osteoarthritis in Clinical Practice. Arthritis Care Res (Hoboken) 2017;69:1287-96.

16. Belk JW, Kraeutler MJ, Houck DA, et al. Platelet-Rich Plasma Versus Hyaluronic Acid for Knee Osteoarthritis: A Systematic Review and Meta-analysis of Randomized Controlled Trials. Am J Sports Med 2021;49:249-60.

17. Di Martino A, Di Matteo B, Papio T, et al. PlateletRich Plasma Versus Hyaluronic Acid Injections for the Treatment of Knee Osteoarthritis: Results at 5 Years of a Double-Blind, Randomized Controlled Trial. Am J Sports Med 2019;47:347-54.

18. Maheu E, Bannuru RR, Herrero-Beaumont G, et al. Why we should definitely include intra-articular hyaluronic acid as a therapeutic option in the management of knee osteoarthritis: Results of an extensive critical literature review. Semin Arthritis Rheum 2019;48:563-72.

19. Stitik TP, Issac SM, Modi S, et al. Effectiveness of 3 Weekly Injections Compared With 5 Weekly Injections of Intra-Articular Sodium Hyaluronate on Pain Relief of Knee Osteoarthritis or 3 Weekly Injections of Other Hyaluronan Products: A Systematic Review and MetaAnalysis. Arch Phys Med Rehabil 2017;98:1042-50.

20. Tammachote N, Kanitnate S, Yakumpor T, et al. IntraArticular, Single-Shot Hylan G-F 20 Hyaluronic Acid Injection Compared with Corticosteroid in Knee Osteoarthritis: A Double-Blind, Randomized Controlled Trial. J Bone Joint Surg Am 2016;98:885-92. 
21. Ha CW, Park YB, Choi CH, et al. Efficacy and safety of single injection of cross-linked sodium hyaluronate vs. three injections of high molecular weight sodium hyaluronate for osteoarthritis of the knee: a double-blind,

Cite this article as: Yang X, Liang W, Li J, Liu P. A metaanalysis and systematic review of the therapeutic effects of arthroscopy combined with intra-articular injection of sodium hyaluronate in the treatment of knee osteoarthritis. Ann Palliat Med 2021;10(9):9859-9869. doi: 10.21037/apm-21-2145 randomized, multi-center, non-inferiority study. BMC Musculoskelet Disord 2017;18:223.

(English Language Editor: J. Reynolds) 